\title{
LA REALIDAD DE LO VIRTUAL Y LA VIRTUALIDAD DE LO REAL EN LA EDUCACIÓN
}

"Todo eso no puede decirse, pero el hombre está para insistir en decirlo: el poeta, en todo caso, el pintor y a veces el loco,,

Julio Cortazar.

La vuelta al dia en ochenta mundos.

\section{Introducción}

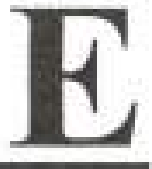

Luis Fernando Correa Calle

Decano Facultad Ingenieria en Computación

Universidad Aüónoma de Manizales

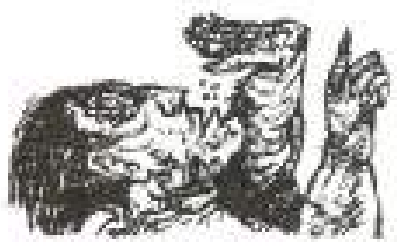

n las últimas décadas, y particularmente en los años transcurridos de la actual, los avances tecnológicos alcanzados en los campos de la informática y las comunicaciones, han despertado un entusiasmo inusitado y un afán por obtener provecho de aquellos, en todos los sectores del quehacer humano.

Poner en duda la utilidad de la tecnologia informática en su estado actual, seria un absurdo, como lo seria también desconocer su capacidad de transformación de la realidad social, económica y cultural. En cuanto al primer aspecto, no es posible negar que hoy día, gracias a esta tecnologia, existen grandes facilidades para la realización de lo que en otro tiempo (en un pasado bastante cercano), representaban disperdiosas tareas, o simplemente per- 
tenecian al mundo de la ficción. En cuanto al segundo aspecto, el de su capacidad de transformación, es claro que su aplicación ha inundado todo el entorno mas inmediato de las personas provocando cambios en sus costumbres y tradiciones, sus prionidades y formas de realizar diferentes actividades, "desplazando el concepto de realidad" . Sin embargo, la aceptación de estos hechos no debe implicar, por ningún motivo. la aplicación irreflexiva de la tecnologia por si misma, sin que medie una evaluación de su potencial frente a una realidad especifica y un "rediseño" de los métodos y formas de intervenir dicha realidad. Esta advertencia se hace considerando que usualmente en cada una de las áreas en que se ha dado aplicación de esta tecnologia. se hacomenzado por emplear los instrumentos tecnológicos en actividades especificas, como sustitutos de personas, pero obedeciendo al tradicional modelo de efectuar las labores. Este es: quema de aplicación de la tecnologia informática no permite aprovechar su verdadero potencial, y en ocasiones conduce a que el poco beneficio obtenido por su uso, se vea opacado por los problemas o los costos que se derivande él.

En el mundo empresarial, existe una preocupacion permanente por obtener el maximo provecho de las innovaciones y por lograr que su uso se traduzca en eficiencia y efectividad en el menor pla- zo posible, por esto muchas empresas han comprendido que los avances tecnológicos en la informutica y las telecomunicaciones conducen a cambios radicales en el "ser" y el modo de "hacer" de las organizaciones, y no simplemente a ayudar a hacer de manera mas sencilla actividades convencionales,

En la educación. donde el tiempo parece transcurrir a un ritmo menos apresurado que en las empresas, y donde parece no existir un interés por los conceptos de eficizncia, eficacia y efectividad (al menos en los procesos educativos mismos; porque en los procesos administrativos si se marnifiesta este interes, aunque aislado de su funcion esencial), se siguen haciendo moderados intentos de aprovechamiento de los avances tecnológicos. Un ejemplo de esto es el empleo que se hace, para algunos temas especificos de aplicaciones de usoftware educativon como tutores, simuladores y enirenadores, incluyéndolos dentro de las actividades de unas pocas asignaturas, obedeciendo mas a su disponibilidad que a criterios pedagógicos. «Si las empresas de hoy no asumen un planteamiento de sus sisternas informaticos como Sistemas de Información (SI), es decir. más allá de un mero software de soporte de funciones administrativas. y no aprovechan su verdadero potencial como generadores de ventajas competitivas desde una pers. pectiva de apoyo a la estrategia

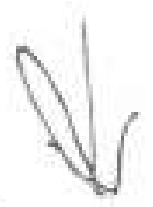

En el mundo empresa-

rial, existe una preocupación permanente por obtener el máximo provecho de las innovaciones y por lograr que su uso se traduzca en eficiencia y efectividad en el menor plazo posible, por esto muchas empresas han comprendido que los avances tecnológicos en la informática y las telecomunicaciones conducen a cambios radicales en el "ser" y el modo de "hacer"... 
de la organización, sin duda șu posicionamiento en el sector se verá perjudicado con el tiempow?

Esta situación de la educación en contraposicion con las organizaciones empresariales, en particular. se ve reflejada tambièn en su lentá adaptación frente a las necesidades de la sociedad, en un ambito más general como lo advierte de manera contundente Julian De Zubiria: aLa sociedad ha cambiado y ya la escuela actual no responde a sus expectativass' , advirtiendo que el cambio experimentado por la sociedad no ha sido motivado exclusivamente por las fuerzas del cambio tecnológico sino tambièn por las fuerzas sociales mismas.

En alguna discusión sobre el estancamiento de la educación frente a la evolución de su entorno social, se escuchó la siguiente reflexion: "Si alguno de nuestros antepasados de por lo menos dos generaciones hacia atrás, tuviera la oportunidad de presenciar el mundo actual, se sorprenderia bastante observando todos los cambios sufridos por la socledad, sus modos de hacer y sus medios, quiza uno de los pocos lugares que hallaria igual seria el aula de clase, donde encontraria a un profesor dictando una clase de la misma manera en que se hacia en su ticmpon. Porque a pesar de los cambios de la sociedad, de sus necesidades y de sus intereses, y de la evolución de los medios e instrumentos que hacen parte de su hacer cotidiano, la educacion se

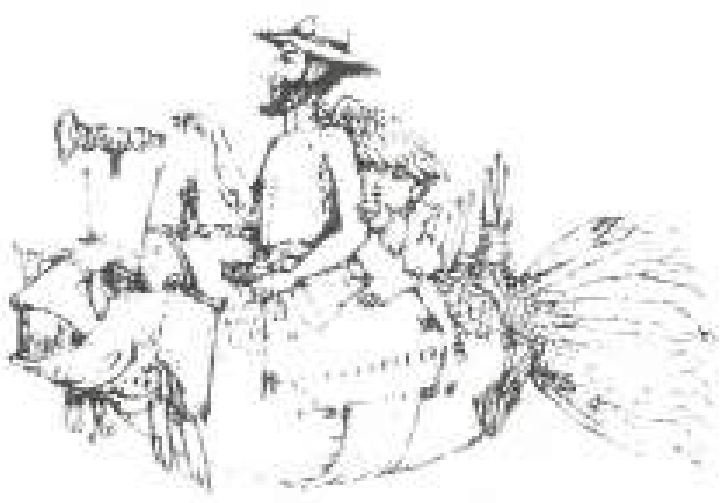

ha quedado anclada en el viejo esquema "transmisionista" en el que se da prioridad a la memorización de información por encima del pensamiento. No obstante se pretende evidenciar su evolucion mediante el uso marginal de tecnologia y la inserción de términos acuñados en otras instancias pero que no corresponden a los moderados y poco substanciales cambios asumidos. El término evirtualy es uno de los que han sido acogidos con más entusiasmo, pretendiendo con él reflejar una verdadera revolución educativa, pero que en la práctica solo consiste en el uso de sofisticados sistemas de telecomunicaciones para sustituir la presencia del docente magistral por una pantalla de televisión y facilitar la realización de evaluaciones, en consideración de la distancia fisica existente entre el docente y el estudiante.

En lo que corresponde al uso de los avances tecnológicos, se puede decir que la educación se permite lujos inadmisibles, en la medida que asume los costos adicionales que su utilizacion encierra, sin exigir de ella un incremento de los indicadores de calidad, cobertura e impacto en el desarrollo social. Parece ser que se privilegia la innovación por si misma. o mas bien la knovedad como elemento atractivo para el cliente potencial, y es por eso que los elementos tecnológicos se *insertanw dentro del modelo educativo tradicional, sustituyendo roles y funciones desempeñados habitualimente por otros actores y elementos, sin que se realice un replanteamiento del modelo y sin que medie interés alguno de solución de la problemática de la educación tradicional (altamente cuestionada), o una reflexión acerca de las necesidades explicitas de la sociedad a la que pertenece. Esta posición es aún más criticable cuando se presenta en un pais inmerso dentro de la problemática del "tercer mundo", donde se suman a la deficiencia de calidad, otros problemas educativos tales como la poca cobertura, la falta de pertinencia y el incremento gradual de los costos de la educación pública ${ }^{a}$. 


\section{Acerca de lo virtual}

Los últimos desarrollos tecnológicos en el campo de la informatica y las telecomunicaciones han propiciado el uso desmedido del tẻrmino virtuat. Quizass la expresión más utilizada ha sido la de realidad virtual, con ella se quiere hacer referencia al estimulo de los sentidos mediante el uso de dispositivos computacionales (hardware y software) con el fin de provocar en la persona la sensación de una realidad inexistente, es decir. indica la simulación de una realidad mediante un conjunto de artificios facilitados por elementos tecnológicos 5 . Pero son muchas otras las palabras que se han acompañado del vocablo virtual para expresar la sustitucion de elementos físicos por otros cuya existencia sólo se evidencia a través de manifestaciones factuales, tal es el caso de la expresión oficina virtual. la cual hace referencia a la posibilidad de desempeñar las labores y actividades reales que usualmente se efectúan en el lugar denominado oficina, sin que este exista fisicamente como tal', De manera semejante se emplean expresiones como supermercado virtual? . ventanilla virtual ${ }^{n}$, Bolsa de valores virtual ${ }^{\circ}$, entre otras.

Las dos connotaciones dadas al concepto de virtualidad implican el uso de un artificio que simula la existencia de objetos o la ocurrencia de hechos irreales o ficticios, o que posibilita la presencia de personas reales pero distantes, en sitios y momentos en los que suceden eventos reales. Joyanes lo expone del siguiente modo ${ }^{10}$ :

"Podrian clasificarse los mundos virtuales en dos grandes grupos:

Simulacion virtual, que implica sistemas que procuran dar la ilusión más convincente posible de inmersión funcional dentro de un mundo sintético.

Televirtualidad, implica sistemas que representan situaciones reales, alejadas o inaccesibles; son las aplicaciones de telemedicina, teleeducación, aula virtual, casa virtual, etc., agrupadas bajo el rótulo de stelepresenciabs

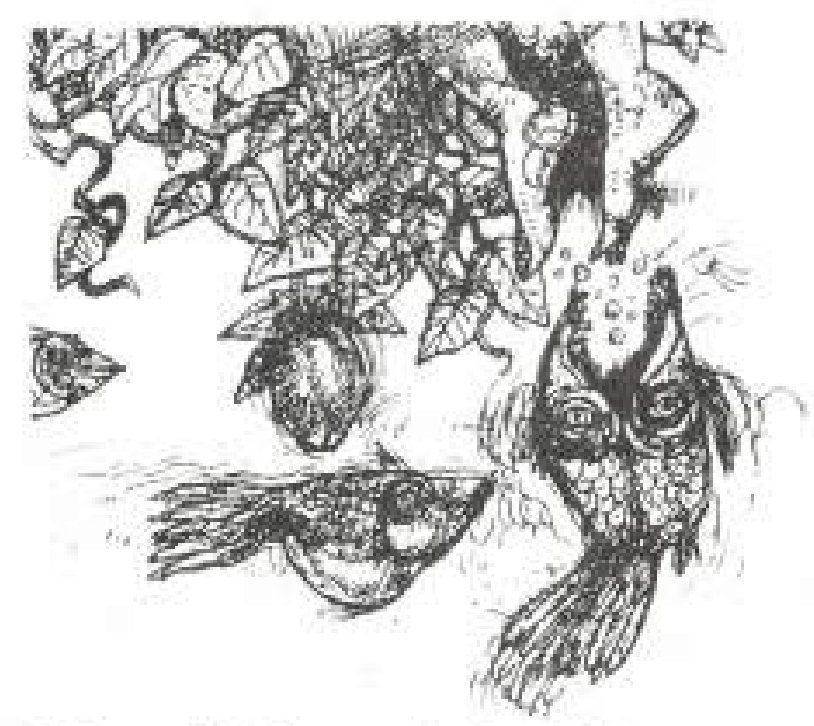

En la actualidad, son varios los artificios o medios tecnológicos que posibilitan la existencia de ambientes virtuales: Las redes de computadores, particularmente Internet con todos sus servicios asociados (teleconferencia, charlas interactivas, correo electrónico, ejecución remota de programas, etc.), los sistemas multimediales, las hiperimägenes (Imágenes que poseen varios niveles distintos de realidad y simulacion, los cuales pueden estar yuxtapuestos. relacionados o fusionados ${ }^{11}$ ), etc.

\section{Lo real de la Educación}

Dentro del proceso evolutivo de la humanidad. la educación ha desempeñado un papel importante como medio de preparaciōn de nuevos individuos para su incorporación al grupo social; ha permitido difundir, preservar y, en ocasiones, transformar el "saber socials (valores, normas de comportamiento, tradiciones, prácticas, etc., de carácter colectivo); y en algunas otras oportunidades, menos afortunadas quizás, ha sido empleada de manera intencional como instrumento de control de la colectividad. 
Si bien en un principio, la capacidad y responsabilidad de la educación podia ser asumida por individuos «sabios», en la medida en que los conocimientos se han hecho más compiejos y abstracios. el proceso educativo ha requerido la intervención de diversas personas «expertasn en àreas especificas, organizadas dentro de lo que son hoy en dia las instituciones educativas ${ }^{12}$. Estas, se encargan de disponer todos los medios fisicos, tecnicos y logisticos. para que el proceso educativo pueda llegar a un conjunto de individuos, orientado por un grupo de expertos temáticos.

La institucionalización de la educación. se ve manifiesta, no en la proliferación de organizaciones dedicadas a ella, si no en el hecho de que social y legalmente solo es avalable (certificable) aguel conocimiento adquirido dentro de alguna institución educativa. Es decir cualquier conocimiento adquirido de forma individual y por interés personal, o por medios no institucionales, sin importar su naturaleza. no es reconocido per se. De alli la gran responsabilidad que la sociedad ha delegado en las instituciones educativas.

Retomando el titulo de este aparte, vale anotar que lo que se denomina aqui $\alpha$ lo real de la educación no es otra cosa que la caracterización de lo que en la práctica ocurre al interior de las institucio- nes educativas dentro del contexto colombiano. Posiblemente algunas de las caracteristicas que aqui se destacan sean comunes a sistemas educativos de otros ámbitos geograficos más amplios o distuntos. pero se podria cacr en generalizaciones peligrosas y poco fundamentadas. Para efectos de la discusión que se quiere plantear, se destacan a continuación algunas de éstas:

Mayor preocupación por la enseñanza que por el aprendizaje: Aunque aparentemente estos dos conceptos presentan una estrecha relaciôn de causa-efecto, no necesariamente la una es consecuencia de la otra. Una frase un poco humoristica puede ayudar a entender esto: kenseñé a silbar a mi perro, pero.... el no aprendión. Las instituciones educutivas mantienen una gran preocupación por poseer y emplear los mejores medios y recursos para apoyar los procesos educativos, de hecho las evaluaciones institucionales sc basan en la cuantificación y cualificación de los métodos y medios utilizados para la enseñanza. Mientras que presentan poca preocupación por el aprendizaje: Cuando uno o varios estudiantes pierden una asignatura, la unica consecuencia de ello es la obligación de repetirla. Adernás, si un estudiante reincide o pierde varias materias. simplemente es expulsado de la institución sin que medie en ella analisis alguno de lo ocurrido ni se indague por las condiciones que pu-

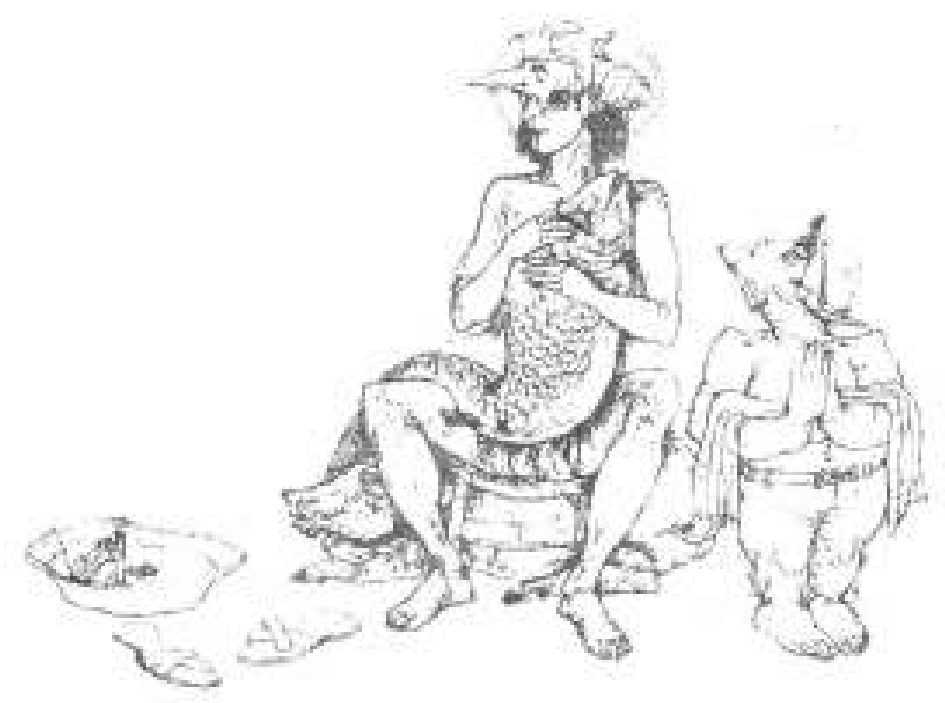

22 Universidad Autónoma de Manizales 
dieron afectar el aprendizaje. es decir se parte de la certeza de que como el proceso de ensenanza se realizo de manera adecuada. el no aprendizaje deja de ser responsabilidad de la institución. Sin embargo el problema es más complejo puesto que la ensenanza es de carácter colectivo, mientras que el aprendizaje es individual: para una institución educativa con un esquema organizativo como el actual, es dificil y costoso administrar procesos educativos que consideren la forma y el ritmo más adecuados de aprendizaje de cada individuo, y en tanto asume que todos los estudiantes deben aprender un conjunto de conocimientos a partir de una ùnica estrategia pedagógica en el mismo tiempo. Frente a este planteamiento hay quienes pretenden emplear como argumento el hecho de que el estudiante tiene la posibilidad de repetit un numero determinado de veces la materia que ha reprobado. y que de esta forma se considera su ritmo de aprendizaje: pero ese planteamiento no es valido puesto que la repeticion de una materia no es otra cosa que la replicación del mismo proceso, generalmente mediante el mismo método de enserianza, abordando cada tema de manera similar y durante periodos de tiempo semejantes, sin tencr en cuenta que es muy posible que algunos temas hayan sido asimilados por el estudiante desde la primera vez, mientras que otros sigan presentando para él mayor dificultad y por tanto requiera ma- yor onentacion y trabajo sobre ellos. En consecuencia, al repetit una materia completa. ei estudiante no solo no tiene mejores condiciones para comprender los temas que no aprendió la primera vez que la curso, si no que adomás puede perder interés y mot. vacion por tener que volver a trabajar sobre los temas que ya sabe

La temporalidad limirada del proceso educativo Las practicas educativas estan delimitadas en tiempo. Los programas curriculares que se ofrecen tienen una duración total fija y se desamollan en fracciones de tiempo fijos icuatrimestres. semestres, años). Esto conduce a que los profesores se apresuren por cumplir con un contenido, sin importar el grado de aprendizaje logrado por los estudiantes ${ }^{13}$. Un grupo de estudiantes debe cursar una materia durante un periodo de tempo fijo, sin que importe la rapidez con que cada individuo la aprenda. La reprobación de un area temática o una materia, implica la repetición durante un periodo fijo de un proceso pre-establecido y pre-programado por la institución sin importar cuàies son los conceptos o la parte del contenido que un estudiante no ha aprendido. Esto significa que dos estudiantes que han reprobado una materia por tener vacios conceptuales diferentes, son sometidos exactamente al mismo proceso (repetición de la materia) y durante un periodo de tiempo igual. El caracter temporal del 
ejercicio educativo está también manifiesto en los horarios de clase. El estudiante está obligado a cumplir con un horario fijo de eventos educativos, el nocumplimiento de estos lo condena al no-reconocimiento de su aprendizajc, segun lo contempla la casi totalidad de los reglamentos institucionales. Cuando un estudiante ha dejado de asistir a un determinado porcentaje (entre el $10 \%$ y el $20 \%$ generalmente) de las actividades académicas programadas por la institución para una asignatura dada, de manera automática y sin importar el conocimiento que el estudiante tenga de la misma, queda marginado de la posibilidad de ser evaluado $y$, por ende, de aprobarla.

\section{La espaciali-} dad restringida del proceso educativo: Desde el momento en que ingresa a una institución educativa y durante cada periodo académico, al estudiante se le informa de manera explicita cuáles son los lugares en los que es posible el aprendizaje $y$ en cuales no. Para todo estudiante $y$, en general para todos los docentes y administradores de las instituciones educativas, es claro que el aula de clase y los laboratorios, son los sitios en los cuales el aprendizaje es posible, dificilmente alguno de ellos puede aceptar que una cafeteria, un bus, una discoteca o un supermercado, son lugares en los cuales es viable aprender, sin embargo estos últimos son lugares reales donde suceden hechos reales y hacen parte de esa realidad para la cual el estudiante se está preparando. Por esa concepción limitada de los espacios de aprendizaje, el estudiante puede estar desperdiciando esa oportunidad de aprender conscientemente de lo que ocurre directamente en la realidad que lo rodea.

\section{- La unidireccionalidad del proceso educa-}

tivo: Las instituciones educativas tienen bien definidos los roles que desempeñan sus actores, unos son los que enseñan, mientras que otros son los que aprenden, es decir, hay unos que saben y otros que no, y la consecuencia es que no puede haberningún otro sentido de flujo del conocimiento. En este es. quema no existe un verdadero espacio de discusión acerca del conocimiento, porque facilmente se puede llegar a conocimientos que yacen fuera del dominio del docente (el que enseña) y este "perderia" autoridad frente a los alumnos (los que aprenden). Este modelo cierra en parte la posibilidad de existencia de espacios investigativos dentro de la institución educativa y limita la posibilidad de aprendizaje de los orientadores de los procesos educativos como de la misma institución, como lo seriala Peter Senge:

"No es accidental que la mayoria de las organizaciones aprendan mal. El modo en que están diseñadas y administradas, el modo en que definen las tareas de la gentey, sobre todo, el modo en que nos han enseñado a pensar e interactuar (no sólo en organizaciones sino en general) crean problemas fundamentales de aprendizaje"i/4

- La enseñanza orientada a solución de problemas: Uno de los medios más utilizados para la enseñanza es la solución de problemas, con estos se busca afianzar y verificar la adquisición del conocimiento por parte de los estudiantes. Los problemas sobre los que se trabaja le son planteados al 
estudiante, tanto cuando se ie enseña como cuando se le evalua. mediante enunciados precisos que contienen toda o casi toda la información que se requiere (en ocasiones se suministra incluso más información de la requerida): pero en la realidad, nunca los problemas aparecen definidos ni acompañados de la información que se requiere para su solucion. por el contrario. es necesario identificarlos y definirlos de manera precisa antes de poder pensar en su solución, y requieren ademas un proceso cuidadoso de búsqueda, obtencion. seleccion y análisis de la información pertinente. siendo estas labores mas arduas y dificiles. debido a que la realidad es bastante compleja y a que estos se encuentran ocultos detrás de complejos sistemas de causa y efecto. Ante la existencia de las restricciones de tiempo y espacio ya mencionadas. se trabaja sobre problemas mucho más simples que los existentes en la realidad. algunos de ellos considerados bajo condiciones ideales. con un alto grado de abstracción y por fuera de su contexto real. Esta situación no solo tiene efecto sobre la pertinencia y aplicabilidad del conocimiento adquindo, st no que tambien limita las posibilidades de plantear, en el interior del proceso educativo. nuevos interrogantes que conduzcan a desarrollar una actitud investigativa en los actores de este proceso.

Secuencialidad temática: La educación se planea como una secuencia ordenada de temas que avanzan de lo simple a lo complejo. De esta manera, el analisis de una situación problematica determinada la realiza el estudianie en forma desagregada y fragmentaria, desde el punto de vista de la asignatura que cursa en un momento dado: "A los alumnos se les enseña por lo general una cosa por vez: Descontextualizadamente y en una secuencia predeterminada. con la falsa expectativa de que esto los llevara a ser lectores y escritores expertos ${ }^{* 15}$. Cuando el estudiante se entrenta luego a un problema real, tiene que esforzarse bastante en articular de nuevo el conocimiento que sus educadores se empeñaron endesagregare independizar Además de esto. la solución de los problemas utilizados en la enseñanza siempre se aborda desde la perspectiva de la disciplina a la cual pertenecen el estudiante y el profesor. pero no se promueve. es más. no se posibilita el trabajo interdisciplinario y en equipo. En el mejor de los casos se admite la realización de trabajos en grupo. pero no se orienta acerca de como trabajar en equi$\mathrm{po}$, ni se hace un seguimiento del proceso del trabajo en equipo:

"El aprendizaje, además. rara vezes cooperativo: por lo general se da por sentado que cada niño trabaja para si mismo, y la cooperación es mirada como una dilapidación de esfuerzos: cuando no como un frau-
En sintesis, las instituciones $y$, especificamente, las aulas en las que se llevan a cabo los procesos educativos actuales, constituyen una realidad virtual que en ocasiones dista bastante de la del entorno vital de los estudiantes, pero que de acuerdo con los esquemas organizativos existentes de las instituciones educativas, es fácil de administrar. 
de. (He descubierto que alumnos de todas las edades, desde la escuela primaria hasta los posgrados, son renuentes a trabajar en equipo en las tareas encomendadas cuando se les indica que lo hagan. Temen que perderán algo,"

\section{La virtualidad de lo real en la educación}

Retomando el concepto de virtual planteado en un aparte anterior, se puede llegar a la conclusión que la educación que se practica actualmente en el pais (aqui denominado como lo real tiene un caracter virtual, en la medida en que se apoya en una serie de artificios que simulan la realidad. Estos artificios son los que conforman el ambiente de aprendizaje existente en un aula de clase: Los problemas sobre los que se trabaja son ficticios: éstos, además, se presentan dentro del aula de clase ya enunciados, definidos y aislados de otros, lo que no ocure en la realidad; el tiempo designado para su solución tiene las restricciones propias de la programación curricular mas no las que se presentan en su contexto real. El espacio en el que se desarrollan los trabajos practicos es una abstracción idealizada de la realidad.

En sintesis, las instituciones y, especificamente.

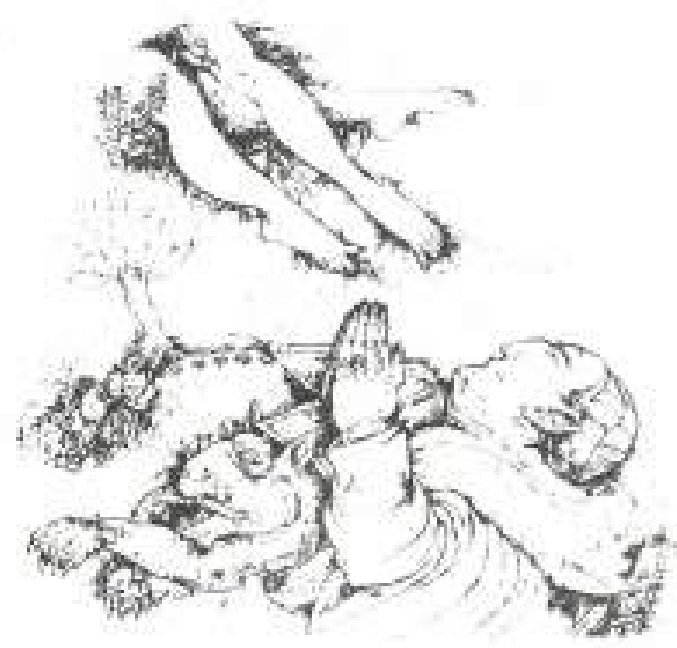

las aulas en las que se llevan a cabo los procesos educativos actuales, constituyen una realidad virtual que en ocasiones dista bastante de la del entorno vital de los estudiantes, pero que de acuerdo con los esquemas organizativos existentes de las instituciones educativas, es facil de administrar.

No tanto por su condición de virtual, si no mas bien pot su distancia con la realidad, el tipo de educación que se viene practicando puede ser poco adecuado por las siguientes razones: No prepara al individuo para la realidad, porque lo hace de manera descontextualizada al margen de la realidad misma. Considera al estudiante como un actor pasivo en el proceso educativo, no le permite tener iniciativa, ni salirse de los limites de la estructura espaciotemporal-curricular en la que se sustenta. No considera los intereses de los estudiantes ni su entorno significativo inmediato. No incentiva actitudes creativas ni la indagación como base de la obtención de nuevo conocimiento.

\section{La realidad de lo virtual en la educación}

Hay una breve historia que se parece bastante a lo que está ocurriendo con el uso de la palabra virtual en la educación: Se trata de dos hombres que se encuentran pescando a la orilla de un rio, de pronto pasa volando un elefante, los hombres un poco sorprendidos se miran pero sin decir nada continuan en su tarea. Más tarde, pasan volando dos elefantes, los hombres se miran pero sin hacer ningü gesto ni hablar. Rato después, pasan cinco elefantes volando. entonces un pescador comenta -deben tener el nido cerca de aquí.

Al igual que en esta historia, el uso de la expresión educación vîrtual se ha ido generalizando sin que haya despertado asombro ni inquietud, y sin una reflexion seria sobre lo que eso significa. Es cierto que el uso de la tecnologia informática y de telecomunicaciones en la educación ha abierto un horizonte promisorio frente a las dificultades o limita- 
ciones que esta última presenta. Las instituciones educativas no consideran la utilización de esta tecnologia como una opción sino como un imperativo. Pero en el afán de éstas por tomar la delantera vecnológica, se ha caido en una simple sustitución de roles sin pensar en una reestructuración completa del proceso educativo que resuelva sus problemas actuales y que aproveche el verdadero potencial de la tecnologia.

En el mundo, existe un número considerable de instituciones educativas que, con el nombre de educación virtual, ofrecen hoy en dia programas educativos bajo esquemas muy convencionales pero con el uso de alta tecnologia, entre ellos se pueden destacar las siguientes ${ }^{17}$ : The New York Institute of Technology, Nova University (Florida), San Diego University, Edison College New Yersey), el Sistema Estatal de Oregon de educación Superior, el sistema de Educación Superior de West Virginia, Open Forum (Inglaterra), la asociación de Televisión Educativa Iberoamericana (ATEI), el Instituto Tecnológico de Monterrey. Todos ellos basan su modelo educativo en videoconferencias en las cuales la presencia del profesor se sustituye por pantallas que presentan su imagen y sus palabras, algunos de ellos cuentan con un sistema de comunicaciones que permiten diálogos interactivos entre el profesor y los estudiantes, sin embargo existe la obligación, por parte de los estudiantes de cumplir el horario en el que se orientan las clases, aunque existe la posibilidad para quienes no pueden asistir a una clase, observar posteriormente el video de la misma. ya sin la opción de la interactividad. Para la comunicación entre estudiantes, profesores y la institución se utiliza el servicio de correo electrónico de Internet o de redes de ărea ancha (WAN) de carăcter público o privado.

Los modelos denominados de educación virtual que hoy en dia se estan utilizando, conservan muchas de las características de la educación tradicional: La temporalidad limitada, la espacialidad resIringida, La unidireccionalidad del proceso edu-

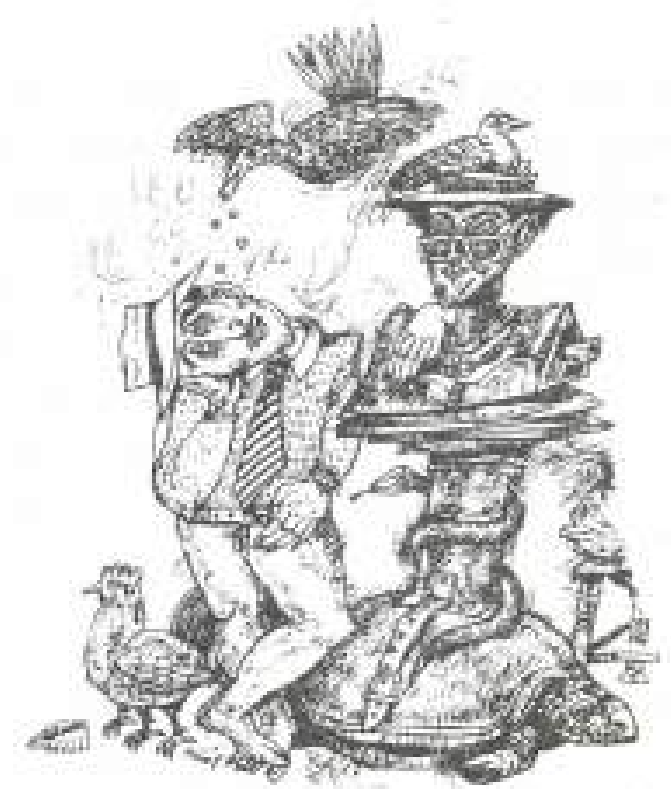

cativo. la enseñanza orientada a la solución de problemas la secuencialidad remática y, además. mantienen el mismo caràcter virtual de la educación tradicional señalado anteriomente. De manera equivoca. en estos modelos se denomina aula virtual a un espacio físico completamente real y al cual deben asistir los estudiantes en un horario preestablecido, alli lo realmente virtual es el profesor, de quien se logra una presencia a través de medios tecnológicos.

El concepto de Institución educativa virtual (ya sea Colegio virtual o Universidad virtual) podria ser válido, siempre y cuando hiciera referencia a una institución sin una existencia física real (o por lo menos no con un campus como existe hoy en dia), pero capaz de generar procesos educativos reales y efectivos. Para alcanzar tal carácter se requiere construir y validar un modelo pedagógico adecuado, que se apoye en una estructura completa de los medios tecnológicos en los que se soportariay, diseñar como complemento un modelo administrativo propio.

\section{Conclusión}

El estado del arte de la tecnologia informática y 
de comunicaciones, ofrece instrumentos de mucha utilidad para enfrentar la problemática actual de la educación. Sin embargo. su utilización dentro de los esquemas educativos actuales, no permite el aprovechamiento real de todo su potencial. ni la solución de los problemas existentes. Muchas instituciones educativas han mostrado más interés en ser pioneros en el uso de la tecnologia, que en investigar acerca de como emplearla de la mejor manera dentro del proceso educativo.

En consecuencia, se hace indispensable la elaboración de propuestas concretas de modelos pedagógicos articulados con los modelos administrativos correspondientes de instituciones educativas. que permitan un mayor aprovechamiento de los recursos tecnológicos y den solución a la problemática de la educación. Los modelos propuestos deben someterse a investigaciones rigurosas pero ágiles que permitan verificar su validez y aplicabilidad en nuestro medio.

JOYANES AGUILAR. Luis. Cibersociedad: Los retos sociales ante un nuevo mundo digital. Madrid. McGraw-Hill. 1997.p.3.

${ }^{2}$ GIL PECHUAN, Ignacio. Sistemas y tecnologias de información para la gestión. Madrid, MeGraw-HilL.1997. p. 19.

'DE ZUBIRIA S. Julian. Los Modelos Pedagógicos. Santafe de Bogota, D.C., FAMDI, 1994, p. 97.

${ }^{4}$ ROSENTHAL, Gert. Algunas dimensiones de la educación para la democracia en el pensamiento de ta CEPAL Internet : www eclac clespanol/portada ASUNCIONESP huml. 1997,p.2

GATES, Bill Camino al futuro. Santafé de Bogota

D.C.: McGraw-Hill lnteramericana, 1995, p. 132-135.

"JOYANES, Luis, op. cit.. 1997, p. 9.10

Ibid. p. I0.

"INTERNET LA ventanilla virtual En: Clase Emipresarial No: 7 7 mayo 1997k, p. 58-61

- EMPRESA VIRTUAL la bolsa en casa. En Clase Empresarial. No. 39 (septiembre 1996): p. 62-63.

"JOYANES, Luis. op cit., 1997, p, 80.

' lbid.p. 82

13 SAVATER. Femando. El valor de educar. Santafe de Bogotà D.C. Ariel S.A..1991, p.41-46.

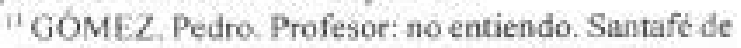
Bogotá D.C. Universidad de los Andes, 1992, p. 136.

i'SENGE, Peter M. La Quinta Disciplina Barcelona: Juan Granica S.A., 1996, p 29.

"SMITH. Frank. De cómo la educación apostó al caballo equivocado. Buenos Aires: Aique grupo editor S.A. 1994, P. 117

1bid. p. 25

EXPERIENCIAS EN Universidad Virtual. En: Inter. net: 200.21 228 34 uvirtualexperien.bum. (noviembre de $1997)$ 